2019-02-06

\title{
Re-evaluation of the
}

\section{ethylene-dependent and -independent}

pathways in the regulation of floral and

organ abscission

Meir, S

http://hdl.handle.net/10026.1/15052

10.1093/jxb/erz038

Journal of Experimental Botany

Oxford University Press (OUP)

All content in PEARL is protected by copyright law. Author manuscripts are made available in accordance with publisher policies. Please cite only the published version using the details provided on the item record or document. In the absence of an open licence (e.g. Creative Commons), permissions for further reuse of content should be sought from the publisher or author. 


\section{1 eXtra Botany}

3 Insight

4 Re-evaluation of the ethylene-dependent and -independent pathways in the 5 regulation of organ abscission focusing on model plants of flower abscission

8 Shimon Meir ${ }^{*}$, Sonia Philosoph-Hadas ${ }^{1}$, Joseph Riov $^{2}$, Mark L. Tucker ${ }^{3}$, Sara E. Patterson ${ }^{4}$, 9 and Jeremy A. Roberts ${ }^{5}$

$10{ }^{1}$ Department of Postharvest Science of Fresh Produce, Agricultural Research Organization (ARO), 11 The Volcani Center, Rishon LeZion 7505101, Israel

$12{ }^{2}$ The Robert H. Smith Institute of Plant Sciences and Genetics in Agriculture, The Robert H. Smith 13 Faculty of Agriculture, Food and Environment, The Hebrew University of Jerusalem, Rehovot 147610001 , Israel

$15{ }^{3}$ Soybean Genomics and Improvement Lab, Agricultural Research Service, United States 16 Department of Agriculture, Bldg. 006, BARC-West, Beltsville, MD 20705, USA

$17{ }^{4}$ Department of Horticulture, University of Wisconsin-Madison, Madison, Wisconsin 53706, USA

$18{ }^{5}$ Office of the Vice-Chancellor, 18 Portland Villas, Drake Circus, Plymouth, Devon, PL4 8AA, 19 UK

$21 \quad$ E-mail address for each author:

22 Shimon Meir - $\underline{\text { shimonm@ volcani.agri.gov.il }}$

23 Sonia Philosoph-Hadas - vtsoniap@ volcani.agri.gov.il

24 Joseph Riov - joseph.riov@ mail.huji.ac.il

25 Mark L. Tucker - mark.tucker@ars.usda.gov

26 Sara E. Patterson - spatters@ wisc.edu

27 Jeremy A. Roberts - jerry.roberts@plymouth.ac.uk

30 Running title: Ethylene and organ abscission in plants 
$32 *$ Corresponding author details:

33 Dr. Shimon Meir,

34 Dept. of Postharvest Science of Fresh Produce, Agricultural Research Organization (ARO), The

35 Volcani Center, Rishon LeZion 7505101, Israel

36 Tel: 972-3-9683667

37 FAX: 972-3-9683878

38 shimonm@volcani.agri.gov.il

39

40 Date of re-submission:

41 Number of figures: 1

42 Word count:

43 
44 Re-evaluation of the ethylene-dependent and -independent pathways in the

45 regulation of organ abscission focusing on model plants of flower abscission

47 Highlights: Ethylene is the key regulator of organ abscission, while the IDA-HAE-HSL2

48 pathway acts downstream of ethylene signaling. The involvement of the turgor pressure in the

49 execution of abscission is suggested.

ABSTRACT

52 Abscission is a developmental process with important implications for agricultural practices.

53 Ethylene has long been considered as a key regulator of the abscission process. The existence of

54 an ethylene-independent abscission pathway, controlled by the complex of INFLORESCENCE

55 DEFICIENT IN ABSCISSION (IDA) peptide and the HAESA (HAE) and HAESA-like2 (HSL2)

56 kinases, has been proposed, based mainly on observations that organ abscission in ethylene-

57 insensitive mutants was delayed but not inhibited. A recent review on plant organ abscission

58 signaling (Patharkar and Walker, 2018), highlighted the IDA-HAE-HSL2 components as the

59 regulators of organ abscission, while the role of auxin and ethylene in this process was hardly

60 addressed. After a careful analysis of the relevant abscission literature, we propose that the IDA-

61 HAE-HSL2 pathway is essential for the final stages of organ abscission, while ethylene plays a

62 major role in its initiation and progression. We discuss the view that the IDA-HAE-HSL2 pathway

63 is ethylene-independent, and present recent evidence showing that ethylene activates the IDA-

64 HAE-HSL2 complex. We conclude that the ability of an organ to abscise is tightly linked to cell

65 turgidity in the abscission zone, and suggest that lack of cell turgidity might contribute to the failure

66 of floral organ abscission in the ida mutants.

68 Key Words:

69 Abscission induction, abscission zone, Arabidopsis, desiccation, ethylene, flower organs, HAESA,

70 HAESA-like2, IDA, turgor.

71 
Abscission is a natural developmental process, in which subtended plant organs, leaves, flowers, fruits, and branches, separate from the parent plant (Osborne, 1989; Lewis et al., 2006). The abscission process has been described as having four stages (Patterson, 2001; Taylor and Whitelaw, 2001; Roberts et al., 2002; Kim, 2014): A, Differentiation of undifferentiated cells to an anatomically discrete abscission zone (AZ); B, Acquisition of the competence of the AZ cells to respond to abscission signals; $\mathbf{C}$, Activation of the AZ cells by the abscission signals, leading to cell wall loosening by newly synthesized cell wall hydrolyzing enzymes and rounding of the AZ cells, which result in organ separation (execution phase);

D, Trans-differentiation of the retained portion of the $\mathrm{AZ}$ to produce a protective layer.

Ethylene was discovered as a gaseous plant hormone associated with organ abscission following its identification as the active component in illuminating gas, which caused leaf abscission in trees growing along urban streets (Abeles et al., 1992; Bakshi et al., 2015; Chang, 2016). Hall (1952) suggested that auxin and ethylene together play a role in the timing of leaf abscission. Auxin has an important role in Stage B of abscission, as the basipetal polar transport of auxin from the distal organ towards the AZ renders it insensitive to ethylene, thereby delaying or preventing abscission. Auxin depletion is a process occurring in the initial stage of abscission, leading finally to organ abscission (reviewed by Meir et al., 2015). Since the above mentioned early discoveries, a substantial body of evidence has been published supporting the role of ethylene as a key regulator of abscission (Jackson and Osborn, 1970; Addicott, 1982; Abeles et al., 1992; Taylor and Whitelaw, 2001; Brown, 2006; Meir et al., 2010). Flower organ abscission in operating through increased ethylene production in flower organs (O'Neill, 1997). abscission in agricultural practices. For example, ethylene releasing compounds are used for thinning of flowers and fruitlets, while ethylene inhibitors are used for inhibiting abscission of flowers, leaves, fruitlets, and fruits (Cooper et al., 1968; van Doorn and Stead, 1997; Michaeli et al., 1999; van Doorn, 2002; Blankenship and Dole, 2003; Malik et al., 2003; Beno-Moualem et

102 Goldental-Cohen et al., 2017). 
The discovery that floral organ abscission in Arabidopsis was only delayed but not prevented in ethylene-insensitive mutants, led to the suggestion that developmental pathways independent of ethylene might contribute to floral organ abscission in this model plant (Bleecker and Patterson, 1997; Butenko et al., 2003; Patterson and Bleecker, 2004). Organ abscission of flower petals, sepals, and stamens in the ethylene-insensitive Arabidopsis mutants occurred in flowers at post anthesis positions (P) 10-15, while in the wild type (WT) abscission occurred at P6-P8.

Two leucine-rich repeat receptor-like kinases (RLKs), HAESA (HAE) and HAESA-like2 (HSL2), and the INFLORESCENCE DEFICIENT IN ABSCISSION (IDA) peptide ligand, were 112 found to be essential for Arabidopsis floral organ abscission (Jinn et al., 2000; Butenko et al., 113 2003, 2006). Additionally, an Arabidopsis ida mutant in which the floral organs did not abscise 114 throughout the development of the siliques was reported, and overexpression of IDA using the 35S 115 promoter enhanced floral organ abscission (Stenvik et al., 2006). The ida plants are ethylene116 sensitive as indicated by the observations that seedlings of the mutant showed the same triple 117 response as WT seedlings, and mature ida plants exhibited enhanced leaf and flower senescence 118 in response to ethylene (Butenko et al., 2003). However, flower organ abscission did not occur in 119 the ethylene-treated ida mutant. These observations led to the suggestion that the IDA-HAE-HSL2 120 complex contributes to an ethylene-independent abscission pathway (Jinn et al., 2000; Butenko et 121 al., 2003; Butenko et al., 2006). The involvement of orthologs of IDA, HAE, and HSL2 in the 122 abscission process has been demonstrated in other plant species (Tucker and Yang, 2012; Stø et 123 al., 2015; Wilmowicz et al., 2018). Moreover, the IDA orthologs from citrus (CitIDA3) (Estornell 124 et al., 2015) and litchi (LcIDL1) (Ying et al., 2016) were shown to be expressed during abscission, 125 and their expression in Arabidopsis accelerated floral organ abscission. In addition, expressing the 126 two IDA orthologs in ida mutants completely restored the abscission ability of the floral organs.

127 Genetic investigations demonstrated that once RLKs are activated by IDA, the RLKs relay 128 a signal through a Mitogen-Activated Protein (MAP) kinase cascade, that ultimately activates 129 KNOTTED1-LIKE HOMEOBOX (KNOX) transcription factors. These factors are suggested to 130 induce the transcription of cell wall remodeling and degrading enzymes responsible for the cell 131 separation process and the subsequent abscission of organs (Cho et al., 2008; Niederhuth et al., 132 2013). Recent reviews on organ abscission signaling in plants (Meng et al., 2016; Patharkar and 133 Walker, 2018) further developed this model, focusing on the cell separation stage, and suggested 
134 that the IDA-HAE-HSL2 are the signaling inducers of organ abscission. This model is supported 135 by the ectopic abscission at branch points and pedicels, in which there are vestigial AZ expressing 136 HAE and HSL2, resulting from overexpression of IDA (Butenko et al., 2006; Stenvik et al., 2006;

137 Stø et al., 2015). The model focuses on factors downstream of IDA/ HAE/HSL, and does not 138 address the role of ethylene, auxin, and other plant hormones in the abscission process.

In the present article, we review the related literature and present evidence that, although IDA is an essential abscission factor, the IDA signaling pathway acts in the last stages of the abscission process rather than in the initiation stage, and serves as a signal to coordinate organ separation and post-abscission events. We also suggest that the spatial expression of the AtIDA

143 gene in the Arabidopsis AZ is regulated by ethylene, as ethylene was found to upregulate the IDA 144 orthologs in several plant species in which these genes were examined (Stø et al., 2015; Ying et $145 a l ., 2016)$. These findings bring into question the hypothesis that the regulation of the IDA-HAE146 HSL2 pathway is ethylene-independent, and suggest that the IDA-HAE-HSL2 pathway acts 147 downstream of ethylene signaling. Finally, the genes coding for cell wall degrading enzymes were 148 upregulated in the $\mathrm{AZ}$ of the ida and hae hsl2 plant similar to their upregulation in the WT 149 (Niederhuth et al., 2013). Based on these observations, we discuss the role of the IDA-HAE-HSL2 150 pathway in inducing the expression of genes coding for cell wall degrading enzymes necessary to 151 fully complete the organ abscission process. We also discuss the importance of cell turgidity in the 152 AZ cells to enable a normal abscission execution. Accordingly, we suggest that the inhibition of 153 floral organ abscission in the ida mutants may result from the loss of cell turgor pressure due to 154 desiccation, and not solely resulting from a reduced upregulation of few cell wall degrading or modifying enzymes as compared to the WT. Taken together, we reassert the role of ethylene as a 156 key regulator of organ abscission, and integrate its role in relation to the IDA-HAE-HSL2 complex 157 and other factors, into a modified abscission model that differs from the models that were recently published (Meng et al., 2016; Patharkar and Walker, 2018).

\section{The IDA-HAE-HSL2 complex does not initiate organ abscission in Arabidopsis}

The decrease in petal breakstrength (a measure of the force required to pull a petal off the 163 wall degradation, which finally leads to separation of the petals from the flower. Arabidopsis 164 flowers develop along a raceme, in which flower P1 is defined when petals are first visible at the 
165 top of the inflorescence. In Arabidopsis WT, the flower petals fully abscise between P6 and P8, 166 shortly after anthesis. In addition, there is evidence showing that the petal abscission process, 167 expressed in terms of decreased petal breakstrength, starts in the WT prior to the detection of the 168 upregulation of IDA gene expression in the AZ. Thus, a significant decrease in the petal 169 breakstrength in the WT was reported to occur at flower P4 (Patterson and Bleecker, 2004; 170 Butenko et al., 2003; Wei et al., 2010; Chen et al., 2011), whereas pIDA::GUS expression was 171 first detected at P5 (Butenko et al., 2003, 2006; Cai and Lashbrook, 2008).

172 If IDA initiates the abscission process, it is expected that the inhibition of the decrease in 173 breakstrength in the ida mutant would occur starting from the initial stage of abscission. However, 174 in both the WT and ida mutant, the petal breakstrength decreased by about 85-90\% at P10 relative 175 to the initial breakstrength at P2 during flower development. Only beyond this position (P10) there was a phenotype in ida in which the breakstrength increased and the petal separation did not occur

177 (Butenko et al., 2003; Stenvik et al., 2008, Shi et al., 2011). Similarly, the petal breakstrength in 178 the hae hsl2 mutant decreased by about $65 \%$ of the initial breakstrength up to flower P10 (Cho et 179 al., 2008; Stenvik et al., 2008; Shi et al., 2011). Nonetheless, there was a delay in the decrease of 180 petal breakstrength in the ida and hae hsl2 mutants from flower P6 up to P10 (Butenko et al., 2003; 181 Liu et al., 2013), which suggested that IDA and HAE/HSL2 are participating in the cell wall 182 loosening process. These observations of breakstrength changes indicate that, similar to the WT, 183 abscission in the mutants is initiated and leads to the activation of cell wall degradation at the early 184 flower positions. The above data suggest, that even though the IDA-HAE-HSL2 complex has a 185 role in the regulation of a subset of several genes involved in the actual cell separation stage, the 186 inhibition of petal abscission in the ida and hae hsl2 mutants cannot be fully explained by the 187 inability of the IDA-HAE-HSL2 complex to coordinate the production or secretion of proteins that 188 contribute to cell wall loosening.

189 Based on the above observations it can be concluded that the IDA-HAE-HSL2 complex 190 is not involved in the actual induction of the organ abscission processes in the AZ, as recently 191 suggested (Meng et al., 2016; Patharkar and Walker, 2018), but it regulates later stages of the 192 process.

193

194 A rapid desiccation of the petals may contribute to the inhibition of floral organ abscission 195 in the ida mutants 
The execution of Stage $\mathbf{C}$ of the abscission process in Arabidopsis is characterized by anatomical changes, including dissolution of the middle lamella and loosening of the cell wall.

198 These changes coincide with elongation and increased volume of the AZ cells at the fracture plane, 199 resulting in their rounded shape observed using scanning electron microscopy (Bleecker and 200 Patterson, 1997; Patterson and Bleecker, 2004). It was previously shown that water movement 201 from mature cells to growing cells is driven by the turgor pressure (Lockhart, 1965), which is 202 essential for elongation and increased cell volume (Lockhart, 1965; Ray et al., 1972; Braidwood 203 et al., 2014). Thus, the cell enlargement process cannot occur in desiccated flower organs or leaves. 204 Scanning electron micrographs, taken during abscission of Arabidopsis flower petals, revealed that 205 rounded cells could be observed in the AZ of the WT at the flower P7-P12 (Bleecker and Patterson, 206 1997; Butenko et al., 2003). In contrast, a flattened fracture plane was detected in the AZ cells at 207 similar flower positions (P8-P12) in the ida mutant, in which the flower petals, which never 208 abscise, started to desiccate and wilt from flower P8 or P10 onwards (Butenko et al., 2003; Cho et 209 al., 2008; Gonzalez-Carranza et al., 2012). The presence of a flattened fracture plane in the AZ 210 cells indicates a partial dissolution of the middle lamella and a lack of turgor pressure. In contrast 211 to the desiccated flower petals of the Arabidopsis ida mutant that remain attached to the 212 inflorescence, in the ethylene-insensitive etrl-1 mutant, turgid and vivid floral organs were present 213 until just before abscission, which finally occurred at flower P11-P15 in various Arabidopsis 214 accessions (Bleecker and Patterson, 1997; Patterson, 2001; Butenko et al., 2003; Patterson and 215 Bleecker, 2004). Similar to the etrl-1 mutant, the petals were turgid when they abscised in another 216 ethylene-insensitive mutant, ein2-1, and also in the delayed abscission mutants (dabs). In addition, 217 scanning electron micrographs of all these mutants showed a similar abscission morphology to 218 that in the WT, namely the occurrence of rounded cells in the AZ (Patterson and Bleecker, 2004; 219 Kim et al., 2013). It is interesting to note, that early screenings performed to identify delayed 220 abscission mutants in the Wisconsin T-DNA collection, revealed that these were actually mutants 221 associated with stress and desiccation, which caused a delayed abscission phenotype (Patterson, 222 personal communication). These observations indicate that the ability of an organ to abscise is also 223 associated with the turgidity of the tissues and the formation of enlarged rounded cells in the AZ. 224 Therefore, it is proposed that loss of turgor pressure in the AZ cells in the ida mutant may 225 significantly contribute to the failure of petal abscission in this mutant.

226 IDA is most likely involved in a pathway controlling the final separation stages in the 
abscission process. This could be a negative signaling pathway inhibiting a repair process induced 228 by the initial dissolution of the middle lamella, or a positive signaling pathway governing the final separation. Interestingly, in plants overexpressing IDA in the floral organ $\mathrm{AZ}$, separation occurred at the flower P4, and a significantly greater number of rounded cells was observed compared to the WT (Stenvik et al., 2006). In the flower positions subsequent to P4, the AZ cells gradually enlarged, and from the flower P6 onwards the AZ was covered with a white substance. These observations further strengthen the relationship between IDA and AZ cell growth leading to the phenotype of enlarged rounded cells that are necessary for separation (Stenvik et al., 2006), and further support our contention that IDA serves as a signal for post-abscission events.

Recently, it was shown that petal desiccation in the ida-2 mutant started at P5, and wilted petals were observed at P8, with no detection of petal abscission, whereas in the WT (Col) the turgid petals abscised at P9 (Ying et al., 2016). In addition, further corroboration of the need for turgid cells during abscission was observed in transgenic Arabidopsis plants expressing LcIDL1 isolated from litchi, in which the $L C I D L 1$ transcript was expressed with the native Arabidopsis IDA promoter in the $i d a-2$ mutant. In these transgenic plants, floral organ abscission was completely restored, and the fast desiccation observed in the ida-2 mutant was prevented (Ying et al., 2016).

An additional example of the strong association between the turgor pressure and abscission of Arabidopsis flower petals is based on the results reported by Basu et al. (2013), who demonstrated that a functional IAA signaling pathway is necessary for abscission to occur. However, unexpected results were obtained when they used a trans-activation approach with the gain-of-function $A X R 3-1$ gene to suppress auxin responses specifically in the floral organ AZ cells. Floral organ abscission was inhibited in the AXR3-1 plants, even though the AXR3-1 protein acts to repress auxin signaling by increasing the stability of the Aux/IAA proteins. These unexpected 250 results could be explained by the observations that the AXR3-1 plants exhibited visual signs of 251 flower senescence, desiccation, and wilting at P8, the flower position in which abscission occurs 252 in the WT. This result supports the hypothesis that desiccation of the AZ cells may inhibit cell 253 separation.

\section{Is the IDA-HAE-HSL2 pathway ethylene-independent?}

It was originally proposed that the IDA gene controls an ethylene-independent pathway of floral organ abscission (Butenko et al., 2003). However, there is evidence that the expression of 
the IDA gene in the AZ of Arabidopsis flower organs is promoted by ethylene, but ethylene may 259 not be essential for the continuity of its expression during abscission (Butenko et al., 2006). Thus, in IDA::GUS plants the onset of the expression in the filament, petal, and sepal AZ in the WT could be detected from the flower P5 and onwards, while in the etr1-1 mutant the signal was not evident in the separating cells even at the flower P8 and P9. Furthermore, wounding of flowers at $\mathrm{P} 1$, in which IDA was not yet expressed, induced expression of the IDA::GUS in the flower petal $\mathrm{AZ} 8 \mathrm{~h}$ after wounding. Increased expression of this gene in the AZ was correlated with the severity of the organ injury (Butenko et al., 2006). The possibility that the wounding effect on IDA::GUS expression was mediated by ethylene and other stresses has not yet been examined. It should be noted that a recent report (Sundaresan et al., 2015) showed that the ethylene production rate of Arabidopsis flowers was relatively high in the flower P2-P5, and it preceded floral organ abscission, which occurred at the flower P7. Additionally, in the eto4 mutant, which overproduces ethylene, the ethylene production rate was twice as much as that of the WT, and as a result, the flower organs of this mutant abscised at an earlier flower position (P5). These results suggest that the induction of ethylene biosynthesis precedes the floral organ abscission and the up-regulation of the IDA gene, which occurs from P5 (Butenko et al., 2006) in the Arabidopsis flower AZ.

Furthermore, ethylene induced the abscission-specific expression of soybean and tomato IDA-like genes, and treatment of soybean with the ethylene action inhibitor 2,5-norbornadiene, greatly delayed the increase in the IDA-like gene expression (Tucker and Yang, 2012). Ethylene also induced expression of the IDA gene in non-AZ tissues, such as petioles and leaf blades, in 278 soybean (Tucker and Yang, 2012). In addition, a recent report demonstrated that ethylene induced the expression of EgHSL and EgIDA genes in the AZ of oil palm fruits (Stø et al., 2015), and that ethephon induced the expression of the IDA-like genes in the AZ cells of the fruitlet peduncle in litchi (Ying et al., 2016), as well as in the AZ of lupine flowers (Wilmowicz et al., 2018). In all the above studies, the increased expression of the IDA or HSL genes in the AZ was detected early, prior to the onset of abscission, indicating a direct effect of ethylene on IDA expression. These 284 observations cast doubt on the hypothesis that the pathway initiated by IDA is solely ethyleneindependent and suggest that the IDA-HAE-HSL2 pathway is promoted by ethylene, and probably controls the abscission processes downstream of ethylene. Recently, Ying et al. (2016) reached a 287 similar conclusion and suggested that IDA acts downstream of ethylene in an ethylene-dependent abscission pathway. It seems therefore, that the hypothesis that the IDA-HAESA complex is an 
ethylene-independent factor leading to floral abscission should be re-evaluated. Further

290 experiments to examine the effect of IDA overexpression in the etrl mutant background are 291 necessary for clarifying this issue.

292 Another example of the regulation of floral organ abscission in an ethylene-independent 293 manner was also proposed by Wei et al. (2010). They reported that overexpression of the 294 Arabidopsis DNA-binding One Finger (DOF) transcription factor (AtDOF4.7) yielded ethylene295 sensitive plants. Since the AtDOF4.7 lines did not exhibit accelerated floral organ abscission 296 following ethylene treatment, the authors concluded that the DOF overexpression led to abscission 297 failure in an ethylene-independent manner. However, the authors seemingly did not notice that the 298 flowers at P1-P2 had already been wilted in the ethylene-treated AtDOF4.7-overexpressed plants compared to the WT flowers, which had turgid petals at P3 and P4 and abscised in response to the ethylene treatment (Wei et al., 2010). Indeed, a recent report of the same laboratory (Wang et al., 2016) demonstrated that ethylene regulates AtDOF4.7, which is involved in the IDA-mediated floral organ abscission pathway. Therefore, it is quite reasonable to suggest that the lack of abscission in the ethylene-treated $i d a$ or $D O F$-overexpressing plants is also due to a rapid desiccation of the flower organs rather than to ethylene-insensitivity.

The IDA-HAE-HSL2 pathway induces gene expression of the cell wall degrading enzymes in a very limited manner

The proposed model that the IDA-HAE-HSL2 complex is the inducer of organ abscission (Meng et al., 2016; Patharkar and Walker, 2018) relies on the assumption that this complex induces the expression of genes that encode proteins that contribute to cell wall disassembly and ultimately lead to organ separation. However, based on microarray (Liu et al., 2013) and RNAseq data 312 low number of Arabidopsis genes encoding cell wall modifying and defense-related enzymes 313 involved in abscission. Liu et al. (2013) used a microarray analysis to identify genes that were 314 downregulated in both the hae hsl2 and ida-2 mutants, including genes encoding cellulose (CEL) 315 and xyloglucan endotransglycosylase/hydrolases (XTHs). Two XTHs genes and a CEL5 gene 316 related to cell wall remodeling, as well as genes related to cell wall loosening and expansion, were 317 downregulated in both the hae hsl2 and ida-2 mutants at P4-P8 compared to the WT. All these 318 genes belong to a cluster of genes that were upregulated between developmental stages 12 to $15 \mathrm{a}$ 319 15c (clusters 1 and 3 in Cai and Lashbrook, 2008). These genes were also upregulated between 
these stages in the mutants, but to a lower level than in the WT. A comparative analysis of the 321 transcriptomes of the Arabidopsis WT and the hae hsl2 mutant at the developmental stage 15, in 322 which abscission had already occurred in the WT, showed a differential expression of a 323 surprisingly low number of cell wall hydrolase genes (Niederhuth et al. 2013), Thus, only 11 out 324 of 189 annotated hydrolase genes were downregulated in the hae hsl2 mutant compared to the WT, and no evidence was provided that any of these genes was specifically expressed in the AZ. 326 Moreover, these 11 genes were actually upregulated in both the hae hsl2 and ida mutants and the 327 WT between stages 12-15 (Niederhuth et al., 2013), indicating that their upregulation in the mutants occurred without the involvement of the IDA-HAE-HSL2 complex, similar to the upregulation of 1772 other genes. The RNA-seq data in the supplementary files of this article were reformulated and presented by Kim et al. (2015). The analyzed data showed that most of the genes related to cell wall disassembly and formation of the protective layer were similarly expressed in

332 the receptacles of the WT and the hae-hsl2 mutant, and some of these genes were even upregulated in the hae-hsl2 mutant compared to the WT (Kim et al., 2015). The minor delay in the decrease of petal breakstrength obtained in the ida and hae hsl2 compared to the WT (Liu et al., 2013), as well as the reformatted results by Kim et al. (2015), indicate that the final cell separation step controlled by the IDA-HAE-HSL2 pathway is effected by a low number of the cell wall hydrolyzing enzymes. Additionally, downregulation of the $p X T R 6:: G U S$ and $p P G A Z A T:: G U S$ expression patterns in the $\mathrm{AZ}$ of the $i d a$ and/or hae hsl2 mutant inflorescences, including the 11 genes reported above, was also reported by others (Gonzalez-Carranza et al., 2012; Kumpf et al., 2013). The observations that a few genes were downregulated in the mutants may explain the slight delay in the decrease of the petal breakstrength in the $i d a$ and hae hsl2 mutants from flower P6 up to flower P10 342 (Butenko et al., 2003; Cho et al., 2008; Stenvik et al., 2008; Shi et al., 2011; Liu et al., 2013). 343 However, it should be emphasized that the process of cell wall remodeling requires the 344 involvement of many gene families related to cell wall degradation, defense, and development of 345 a boundary layer that are specifically upregulated in the AZ. Numerous such genes were detected 346 in various abscission systems including tomato flowers (Meir et al., 2010; Kim et al., 2015), fruits 347 of apple (Zhu et al., 2011), melon (Corbacho et al., 2013) and olive (Gil-Amado and Gomez348 Jimenez, 2013), fruitlets of litchi (Li et al., 2015) and citrus (Xie et al., 2018), soybean leaves 349 (Kim et al., 2015), and lupine florets (Glazinska et al., 2017). It seems therefore, that the IDA350 HAE-HSL2 pathway appears to regulate only a subset of genes encoding cell wall modifying 
proteins expressed in AZ cells.

\section{Conclusions and perspectives}

354 Based on our analysis of the literature, there is overwhelming evidence that ethylene plays 355 a crucial role in the timing of Arabidopsis floral abscission. We believe that the recent model regarding the ethylene-independent pathway suggested to be governed by IDA-HAE-HSL2 requires further evaluation, particularly the mechanisms by which this signaling complex operates alongside ethylene in regulating organ shedding. The new models of abscission regulation have mostly been derived from genetic approaches based on ethylene-insensitive mutants, that showed delayed abscission phenotypes, and on the non-abscising phenotypes of ida and hae hsl2 mutants. The fact that abscission in ethylene-insensitive mutants, like etrl-1, is delayed but not inhibited suggests, that in these mutants an alternative regulator of abscission can be activated at later stages of development, much after abscission occurrence in the WT. Nevertheless, in Arabidopsis WT plants and most other abscission systems, the increase in ethylene biosynthesis that coincides with auxin depletion, leading to increased sensitivity to ethylene, is the key regulator of the abscission process (Meir et al., 2015). Therefore, further efforts should be devoted to explore the crosstalk between ethylene, IAA, and the IDA-HAE-HSL2 pathway in coordinating organ abscission. A summarizing scheme, which takes into account all the factors mentioned here, illustrates the sequence of events in the abscission process of Arabidopsis plants (Box 1).

Moreover, we suggest that a tight link between the turgor pressure and the 'rounding up' of the AZ cells may play a critical role in the final act of organ shedding. Accordingly, there is a need to reexamine the rate of desiccation of AZ tissues in both the WT and the mutant plants, such as the ida mutant, in which a flattened or broken fracture plane in the AZ cells is detected. One

374 approach that might be of particular interest in this regard is to investigate how the IDA peptide affects the water relations of the AZ cells, and whether IDA has an effect on aquaporins in the AZ cells during the final stages of organ separation.

It seems therefore, that a visible simple process such as abscission of the floral organs of Arabidopsis is clearly a very complex process that involves multiple pathways, the exact interactions of which are yet to be determined, and other un-elucidated pathways. In addition, while the basic involvement of the IDA-HAE-HSL2 pathway in the Arabidopsis abscission process 
382 plants from which they were isolated. For example, overexpression of the litchi LcIDL1 gene 383 caused earlier floral organ abscission in Arabidopsis (Ying et al., 2016), but the role of this gene 384 in litchi is still unknown. Thus, the model of Arabidopsis floral organ abscission (Box 1) cannot 385 be yet applied to other plant species.

\section{References}

Abeles F, Morgan P, Saltveit MJ. 1992. Ethylene in plant biology. Second Ed. San Diego, CA: Academic Press.

Addicott FT. 1982. Abscission. Berkeley: University of California Press.

Agusti J, Merelo P, Cercos M, Tadeo FR, Talon M. 2008. Ethylene-induced differential gene expression during abscission of citrus leaves. Journal of Experimental Botany 59, 2717-2733.

Ascough GD, Nogemane N, Mtshali NP, van Staden J. 2005. Flower abscission: environmental control, internal regulation and physiological responses of plants. South African Journal of Botany 71, 287-301.

Bakshi A, Shemansky JM, Chang C, Binder BM. 2015. History of research on the plant hormone ethylene. Journal of Plant Growth Regulation 34, 809-827.

Basu MM, González-Carranza ZH, Azam-Ali S, Tang S, Shahid AA, Roberts JA. 2013. The manipulation of auxin in the abscission zone cells of Arabidopsis flowers reveals that indoleacetic acid signaling is a prerequisite for organ shedding. Plant Physiology 162, 96-106.

Beno-Moualem D, Gusev L, Dvir O, Pesis E, Meir S, Lichter A. 2004. The effects of ethylene, methyl jasmonate and 1-MCP on abscission of cherry tomatoes from the bunch and expression of endo-1,4- $\beta$-glucanases. Plant Science 167, 499-507.

Blankenship SM, Dole JM. 2003. 1-Methylcyclopropene: a review. Postharvest Biology and Technology 28, 1-25.

Bleecker AB, Patterson SE. 1997. Last exit: Senescence, abscission, and meristem arrest in Arabidopsis. The Plant Cell 9, 1169-1179.

Braidwood L, Breuer C, Sugimoto K. 2014. My body is a cage: mechanisms and modulation of plant cell growth. New Phytologist 201, 388-402.

Brown K. 2006. Ethylene and abscission. Physiologia Plantarum 100, 567-576.

Butenko MA, Patterson SE, Grini PE, Stenvik GE, Amundsen SS, Mandal A, Aalen RB. 
2003. INFLORESCENCE DEFICIENT IN ABSCISSION controls floral organ abscission in Arabidopsis and identifies a novel family of putative ligands in plants. The Plant Cell 15, 22962307.

Butenko MA, Stenvik GE, Alm V, Saether B, Patterson SE, Aalen RB. 2006. Ethylenedependent and -independent pathways controlling floral abscission are revealed to converge using promoter::reporter gene constructs in the ida abscission mutant. Journal of Experimental Botany 57, 3627-3637.

Cai S, Lashbrook CC. 2008. Stamen abscission zone transcriptome profiling reveals new candidates for abscission control: enhanced retention of floral organs in transgenic plants overexpressing Arabidopsis ZINC FINGER PROTEIN2. Plant Physiology 146, 1305-1321.

Chang C. 2016. Q\&A: How do plants respond to ethylene and what is its importance? BMC Biology 14, 7.

Chen M-K, Hsu W-H, Lee P-F, Thiruvengadam M, Chen H-I, Yang C-H. 2011. The MADS box gene, FOREVER YOUNG FLOWER, acts as a repressor controlling floral organ senescence and abscission in Arabidopsis. The Plant Journal 68, 168-185.

Cho SK, Larue CT, Chevalier D, Wang H, Jinn T-L, Zhang S, Walker JC. 2008. Regulation of floral organ abscission in Arabidopsis thaliana. Proceedings of the National Academy of Sciences, USA 105, 15629-15634.

Cooper WC, Rasmussen GK, Rogers BJ. 1968. Control of abscission in Agricultural crops and its physiological basis. Plant Physiology 43, 1560-1576.

Corbacho J, Romojaro F, Pech JC, Latché, Gomez-Jimenez MC. 2013. Transcriptomic events involved in melon mature-fruit abscission comprise the sequential induction of cell-wall degrading genes coupled to a stimulation of endo and exocytosis. PLoS ONE 8, e58363.

Estornell LH, Wildhagen M, Pérez-Amador MA, Talón M, Tadeo FR, Butenko MA. 2015. The IDA peptide controls abscission in Arabidopsis and citrus. Frontiers in Plant Science 6, 1003. doi: 10.3389/fpls.2015.01003

Gil-Amado JA, Gomez-Jimenez MC. 2013. Transcriptome analysis of mature fruit abscission control in olive. Plant \& Cell Physiology 54, 244-269.

Glazinska P, Wojciechowski W, Kulasek M, Glinkowski W, Marciniak K. Klajn N, Kesy J, Kopcewicz J. 2017. De novo transcriptome profiling of flowers, flower pedicels and pods of Lupinus luteus (Yellow Lupine) reveals complex expression changes during organ abscission. 
Frontiers in Plant Science 8, 641. doi: 10.3389/fpls.2017.00641.

Goldental-Cohen S, Burstein C, Biton I, Ben-Sasson S, Sadeh A, Many Y, Doron-Faigenboim A, Zemach H, Mugira Y, Schneider D, Birger R, Meir S, Philosoph-Hadas S, Irihomovitch V, Avidan B, Lavee S, Ben-Ari G. 2017. Ethephon-induced oxidative stress in the olive leaf abscission zone enables development of a selective abscission compound. BMC Plant Biology 17, 87. doi: 10.1186/s 12870-017-1035-1.

Gonzalez-Carranza ZH, Shahid AA, Zhang, Liu Y, Ninsuwan U, Roberts JA. 2012. A novel approach to dissect the abscission process in Arabidopsis. Plant Physiology 160, 1342-1356. doi: 10.1104/pp.112.205955.

Hall. W. 1952. Evidence on the auxin-ethylene balance hypothesis of foliar abscission. Botanical Gazette 113, 310-322.

Jackson MB, Osborne DJ. 1970. Ethylene, the natural regulator of leaf abscission, Nature 225, 1019-1022.

Jinn TL, Stone JM, Walker JC. 2000. HAESA, an Arabidopsis leucine-rich repeat receptor kinase, controls floral organ abscission. Genes \& Development 14, 108-117.

Kim J. 2014. Four shades of detachment: regulation of floral organ abscission. Plant Signaling \& Behavior 9, e976154.

Kim J, Dotson B, Rey C, Lindsey J, Bleecker AB, Binder BM, Patterson SE. 2013. New clothes for the jasmonic acid receptor COI1: delayed abscission, meristem arrest and apical dominance. PLoS ONE 8, e60505.

Kim J, Sundaresan S, Philosoph-Hadas S, Yang R, Meir S, Tucker ML. 2015. Examination of the abscission-associated transcriptomes for soybean, tomato and Arabidopsis highlights the conserved biosynthesis of an extensible extracellular matrix and boundary layer. Frontiers in Plant Science 6, doi: 10.3389/fpls.2015.01109.

Kumpf RP, Shi C-L, Larrieu A, Stø IM, Butenko MA, Péret B, Riiser ES, Bennett MJ, Aalen RB. 2013. Floral organ abscission peptide IDA and its HAE/HSL2 receptors control cell separation during lateral root emergence. Proceedings of the National Academy of Sciences USA 110, 52355240.

Lewis MW, Leslie ME, Liljegren SJ. 2006. Plant separation: 50 ways to leave your mother. Current Opinion in Plant Biology 9, 59-65. 
Li C, Wang Y, Ying P, Ma W, Li J. 2015. Genome-wide digital transcript analysis of putative fruitlet abscission related genes regulated by ethephon in litchi. Frontiers in Plant Science 6, 502. doi: 10.3389/fpls.2015.00502.

Liu B, Butenko MA, Shi C-L, Bolivar JL, Winge P, Stenvik GE, Vie AK, Leslie MF, Brembu T, Kristiansen W, Bones AM, Patterson SF, Liljegren SJ, Aalen RB. 2013. NEVERSHED and INFLORESCENCE DEFICIENT IN ABSCISSION are differentially required for cell expansion and cell separation during floral organ abscission in Arabidopsis thaliana. Journal of Experimental Botany 64, 5345-5357.

Lockhart JA. 1965. An analysis of irreversible plant cell elongation. Journal of Theoretical Biology 8, 264-275.

Malik AU, Agrez V, Singh Z. 2003. Fruitlet abscission of mango in relation to ethylene. Journal of Horticultural Science and Biotechnology 78, 458-462.

Meir S, Philosoph-Hadas S, Sundaresan S, Selvaraj KS, Burd S, Ophir R, Kochanek B, Reid MS, Jiang C-Z, Lers A. 2010. Microarray analysis of the abscission-related transcriptome in the tomato flower abscission zone in response to auxin depletion. Plant Physiology 154, 1929-1956.

Meir S, Sundaresan S, Riov J, Agarwal I, Philosoph-Hadas S. 2015. Role of auxin depletion in abscission control. Stewart Postharvest Review 11, 1-15.

Meng X, Zhou J, Tang J, Li B, de Oliveira MV, Chai J, He P, Shan L. 2016. Ligand-induced receptor-like kinase complex regulates floral organ abscission in Arabidopsis. Cell Reports 14, 1330-1338.

Michaeli R, Philosoph-Hadas S, Riov J, Meir S. 1999. Chilling-induced leaf abscission of Ixora coccinea plants. I. Induction by oxidative stress via increased sensitivity to ethylene. Physiologia Plantarum 107, 166-173.

Niederhuth CE, Patharkar OR, Walker JC. 2013. Transcriptional profiling of the Arabidopsis abscission mutant hae hsl2 by RNA-Seq. BioMed Central Genomics 14, 37-49.

O'Neill SD. 1997. Pollination regulation of flower development. Annual Review of Plant Physiology and Plant Molecular Biology 48, 547-574.

Osborne DJ. 1989. Abscission. Critical Reviews in Plant Sciences 8, 103-129. 
Patharkar OR, Walker JC. 2018. Advances in abscission signaling. Journal of Experimental Botany 69, 733-740.

Patterson SE. 2001. Cutting loose. Abscission and dehiscence in Arabidopsis. Plant Physiology 126, 494-500.

Patterson SE, Bleecker AB. 2004. Ethylene-dependent and -independent processes associated with floral organ abscission in Arabidopsis. Plant Physiology 134, 194-203.

Ray PM, Green PB, Cleland R. 1972. Role of turgor in plant cell growth. Nature 239, 163-164. Roberts JA, Elliott KA, Gonzalez-Carranza ZH. 2002. Abscission, dehiscence, and other cell separation processes. Annual Review of Plant Biology 53, 131-158.

Roberts JA, Gonzalez-Carranza ZH. 2007. Abscission. Encyclopedia of Life Sciences. John Wiley \& Sons, Ltd., 1-8. doi: 10.1002/9780470015902.a0020089.

Shi CL, Stenvik GE, Vie AK, Bones AM, Pautot V, Proveniers M, Aalen RB, Butenko MA. 2011. Arabidopsis class I KNOTTED-like homeobox proteins act downstream in the IDAHAE/HSL2 floral abscission signaling pathway. The Plant Cell 23, 2553-2567.

Stenvik GE, Butenko MA, Urbanowicz BR, Rose JK, Aalen RB. 2006. Overexpression of INFLORESCENCE DEFICIENT IN ABSCISSION activates cell separation in vestigial abscission zones in Arabidopsis. The Plant Cell 18, 1467-1476.

Stenvik GE, Tandstad NM, Guo Y, Shi CL, Kristiansen W, Holmgren A, Clark SE, Aalen RB, Butenko MA. 2008. The EPIP peptide of INFLORESCENCE DEFICIENT IN ABSCISSION is sufficient to induce abscission in Arabidopsis through the receptor-like kinases HAESA and HAESA-LIKE2. The Plant Cell 20, 1805-1817.

Stø IM, Orr RJS, Fooyontphanich K, Jin X, Knutsen JMB, Fischer U, Tranbarger TJ, Nordal I, Aalen RB. 2015. Conservation of the abscission signaling peptide IDA during Angiosperm evolution: withstanding genome duplications and gain and loss of the receptors HAE/HSL2. Frontiers in Plant Science 6, 931. doi: 10.3389/fpls.2015.00931.

Sundaresan S, Philosoph-Hadas S, Riov J, Belausov E, Kochanek B, Tucker ML, Meir S. 2015. Abscission of flowers and floral organs is closely associated with alkalization of the cytosol in the abscission zone cells. Journal of Experimental Botany 66, 1355-1368. dol:10.1093/jxb/eru483.

Taylor JE, Whitelaw CA. 2001. Signals in abscission. New Phytologist 151, 323-340. 
Tucker ML, Yang R. 2012. IDA-like gene expression in soybean and tomato leaf abscission and requirement for a diffusible stelar abscission signal. AoB Plants. doi:10.1093/aobpla/pls035.

van Doorn WG. 2002. Effect of ethylene on flower abscission: A survey. Annals of Botany 89, 689-693.

van Doorn WG, Stead AD. 1997. Abscission of flowers and floral parts. Journal of Experimental Botany 48, 821-837.

Wang GQ, Wei PC, Tan F, Yu M, Zhang A-Y, Qi-Jun Chen QJ, Wang XC. 2016. The Transcription factor AtDOF4.7 is involved in ethylene and IDA-mediated organ abscission in Arabidopsis. Frontiers in Plant Science 7, 863. doi: 10.3389/fpls.2016.00863

Wei PC, Tan F, Gao XQ, Zhang XQ, Wang GQ, Xu H, Li LJ, Chen J, Wang XC. 2010. Overexpression of AtDOF4.7, an Arabidopsis DOF family transcription factor, induces floral organ abscission deficiency in Arabidopsis. Plant Physiology 153, 1031-1045.

Wilmowicz E, Kućko A, Ostrowski M, Panek K. 2018. INFLORESCENCE DEFICIENT IN ABSCISSION-like is an abscission associated and phytohormone-regulated gene in flower separation of Lupinus luteus. Plant Growth Regulation 85, 91-100. doi:org/10.1007/s10725-0180375-7.

Xie R, Ge T, Zhang J, Pan X, Ma Y, Yi S, Zheng Y. 2018. The molecular events of IAA inhibiting citrus fruitlet abscission revealed by digital gene expression profiling. Plant Physiology and Biochemistry 130, 192-204.

Ying P, Li C, Liu X, Xia R, Zhao M, Li J. 2016. Identification and molecular characterization of an IDA-like gene from litchi, LcIDL1, whose ectopic expression promotes floral organ abscission in Arabidopsis. Scientific Reports 15, 6:37135. doi: 10.1038/srep37135.

Zhu H, Dardick CD, Beers EP, Callanhan AM, Xia R, Yuan R. 2011. Transcriptomics of shading-induced and NAA-induced abscission in apple (Malus domestica) reveals a shared pathway involving reduced photosynthesis, alterations in carbohydrate transport and signaling and hormone crosstalk. BMC Plant Biology 11, 138. doi: 10.1186/1471-2229-11-138. 


\section{Figure legends}

Figure 1. A simplified schematic model depicting the sequence of events in AZ formation and flower organ abscission in Arabidopsis. The abscission process is depicted in four stages. A, Differentiation of the abscission zone (AZ). B, Acquisition of the competence of the AZ cells to respond to ethylene. C, Activation of the AZ cells by ethylene and execution phase, synthesis of cell wall hydrolyzing enzymes, and water uptake by AZ cells to enable their growth and rounded phenotype, which lead to organ separation. D, Trans-differentiation of the retained portion of the AZ to produce a protective defense layer. We represent in the model the main stages of the abscission process, highlighting a differentiation between the roles of ethylene and the IDA-HAEHSL2 pathway and the crosstalk (purple overlapping lines) between these two signals. This model delineates the role of ethylene in regulating the initiation and subsequent regulation of the abscission process following auxin depletion, and the regulation by the IDA-HAE-HSL2 signaling pathway at more advanced stages of abscission, when the breakstrength is already in the process of decreasing. This scheme is adapted and updated from Bleecker and Patterson (1997) and Kim et al. (2015). 